\title{
Plasmodium yoelii blood-stage primes macrophage-mediated innate immune response through modulation of toll-like receptor signalling
}

Yong $\mathrm{Fu}^{1}$, Yan Ding ${ }^{1}$, Taoli Zhou ${ }^{1}$, Xiaolan $\mathrm{Fu}^{2}$ and Wenyue $\mathrm{Xu}^{1 *}$

\begin{abstract}
Background: Toll-like receptors (TLRs) signalling is reported to be primed by the infection of human malaria parasite, Plasmodium falciparum. However, little is known about the regulation of macrophages TLR signalling by the infection of lethal or non-lethal strain of rodent malaria parasites.

Methods: BALB/c mice were infected with non-lethal strain Plasmodium yoelii 17XNL or lethal strain P. yoelii 17XL. Peritoneal macrophages were isolated to study its immune response to pRBC lysate, and TLRs (TLR2, TLR4, and TLR9) agonists, and the expression of TLRs and intracellular signalling molecules were also investigated by flow cytometry and semi-quantitive RT-PCR.

Results: The reactivity of peritoneal macrophages from the mice infected with lethal strain $P$. y $17 X \mathrm{~L}$ or non-lethal strain P. y 17XNL were enhanced to pRBC lysate, and TLR2, TLR4, and TLR9 agonists at one, three and five days post-infection. Of all the tested TLRs, only TLR2 was up-regulated on peritoneal macrophages of mice infected with either strain. However, transcription of intracellular signalling molecules MyD88, IRAK-1, and TRAF-6 was significantly up-regulated in peritoneal macrophages from mice infected either with $P$. yoelii $17 \mathrm{XL}$ or $P$. yoelii 17XNL at one, three and five days post-infection. However, the enhanced TLRs response of macrophage from $P$. yoelii $17 X N L-$ infected mice persisted for a much longer time than that from $P$. yoelii 17XL-infected mice.

Conclusion: Both P. yoelii 17XL and 17XNL strains could enhance the response of peritoneal macrophages to pRBC lysate and TLR agonists, through up-regulating the expression of TLR2 and intracellular signalling molecules MyD88, IRAK-1, and TRAF-6. In addition, prolonged high response of macrophage from P. yoelii 17XNL-infected mice might be associated with the more efficiently controlling of $P$. yoelii $17 X N L$ growth in mice at early stage.
\end{abstract}

Keywords: Plasmodium yoelii, Macrophage, Toll-like receptors

\section{Background}

Malaria remains one of the most devastating diseases worldwide, with $\sim 40 \%$ of the population at risk, and 200-300 million new cases each year, resulting in about one million deaths annually [1]. The causative agents of malaria are parasitic protozoa belonging to the genus Plasmodium. Except for the virulence of infected

\footnotetext{
* Correspondence: xuwenyue@gmail.com

'Department of Pathogenic Biology, Third Military Medical University, 30 Gaotanyan Zhengjie, Shapingba District, Chongqing 400038, People's Republic of China

Full list of author information is available at the end of the article
}

malaria parasite, presentation of clinical malaria is mainly dependent on the balance between pro- and anti-inflammatory responses against these parasites. Individuals who exhibit particularly weak immune responses often lead to uncontrolled parasitaemia. Thus, understanding the regulation mechanism of the immune response brought on by infection with Plasmodium parasites will provide us with potential therapeutic approaches for treating infected individuals.

Although adaptive immune effectors, such as Plasmodium-specific $\mathrm{CD}_{4}^{+} \alpha \beta \mathrm{T}$ cells and antibody [2], are mandatory for effective clearance of parasitized red 
blood cells (pRBCs) after infection, the control of parasite growth during the early stage of infection is largely dependent on the innate immune response. Previous study has shown that the primary peak of parasitaemia in T-cell-deficient mice tends to be comparable to that of wild-type mice [3]. It has been reported that NK cellderived IFN- $\gamma$ that contributes to the early control of Plasmodium chabaudi and Plasmodium yoelii infections $[4,5]$. However, a recent study showed that the macrophage-mediated innate immune response, but not IFN- $\gamma$, has a significant role in controlling the primary wave of $P$. yoelii infection [6]. Another study recently reported that a population of $\mathrm{CD} 11 \mathrm{~b}^{\text {high }} \mathrm{Ly} 6 \mathrm{C}^{+}$monocyte migration from bone marrow to the spleen was important for killing of $P$. chabaudi blood stage at early stage [7].

It is well established that macrophages are activated by malaria parasites to release pro-inflammatory cytokines, mainly through toll-like receptors (TLRs). For example, Plasmodium falciparum-derived glycosylphosphatidylinositols (GPI) moieties are known to induce potent TNF responses in macrophages by TLR2, and to a lesser extent TLR4[8,9]. It has also been reported that $P$. falciparum haemozoin, a crystalline by-product of haemoglobin metabolism by malaria parasites, is recognized by TLR9 on macrophages[10], although more recently it has been suggested that instead haemozoin-bound nucleic acids are the true ligand for this receptor $[11,12]$.

TLR signalling is subject to modulation by microorganisms. For instance, it is well known that lipopolysaccharide (LPS), the toxic wall component of Gramnegative bacteria, induces macrophages into a tolerance status by down-regulating TLR4 receptor expression [13], or its association with MyD88, and by IRAK-1 activation [14]. In contrast, infection of $P$. falciparum primes peripheral blood mononuclear cells (PBMC) for TLR signalling by enhancing mitogen-activated protein kinase (MAPK) activation during the early stage [15-17]. However, the infection of $P$. yoelii non-lethal strain 17XNL was reported to induce dendritic cell (DC) TLR tolerance during the late stage [18].

Little is known about the regulation of macrophages TLR signalling by the infection of lethal or non-lethal strain of rodent malaria parasites at the early stage. In the present study, both P. yoelii 17XL and 17XNL strains were found to be able to enhance the response of peritoneal macrophages to $\mathrm{PRBC}$ lysate and TLR agonists, through up-regulating the expression of TLR2 and intracellular signalling molecules MyD88, IRAK-1, and TRAF-6.

\section{Methods}

\section{Mice and plasmodium}

BALB/c mice (specific pathogen free, $\sim 6-8$ week old females) were purchased from the Animal Institute of
Third Military Medical University (Chongqing, China). These studies have been reviewed and approved by the Third Military Medical University Institute of Medical Research Animal Ethics Committee. P. yoelii 17XNL is a non-lethal strain, originally isolated in 1965 from the blood of a wild thicket rat, Thamnomys rutilans [19], and P. yoelii 17XL is a lethal strain cloned from P. yoelii $17 \mathrm{XNL}$, which was suddenly virulent in the laboratory of J. Finerty [20]. Cohorts of 10 mice were infected intraperitoneally with $2 \times 10^{5}$ non-lethal strain $P$. yoelii 17XNL-infected pRBCs or $2 \times 10^{5}$ lethal strain $P$. yoelii $17 \mathrm{XL}$. For control purposes, 10 mice were also infected with $2 \times 10^{5}$ RBCs taken from normal mice (nRBCs).

\section{Reagents and antibody}

Biotin anti-mouse TLR4 (MTS510), TLR2 (6 C2 clone), TLR9 (M9.D6 clone), Biotin rat IgG2a and IgG2b isotype control antibodies, anti-mouse CD16/32 (93 clone), FITC-F4/80 and PE-streptavidin were all purchased from eBioscience (San Diego, CA). TLR2 agonist Pam3CSK4, TLR4 agonist LPS (Escherichia.coli 0111: B4), TLR9 agonist CpG(ODN 1826), and its control CpG were purchased from InvivoGen (San Diego, CA).

\section{Isolation of peritoneal macrophage}

All the mice infected with $P$. yoelii $17 \mathrm{XL}$ or $17 \mathrm{XNL}$, or injected with nRBCs, were administrated with $1 \mathrm{mg} / \mathrm{ml}$ thioglycollate (Sigma, St. Louis, MO, USA) via intraperitoneally three days before killing. Peritoneal exudate cells (PECs) were then extracted and allowed to adhere on tissue culture dishes for two hours, and non-adherent cells were removed. The adherent cells were collected as peritoneal macrophages, and F4/80 expression was analysed using a FACSCalibur flow cytometer with Cell Quest software (Becton Dickinson, Lincoln Park, NJ).

\section{Cytokine detection by ELISA}

Peritoneal macrophage from mice infected with P. yoelii $17 \mathrm{XL}, 17 \mathrm{XNL}$, or nRBCs were cultured in the presence of $1 \times 10^{7} \mathrm{nRBC}$ or pRBC lysate, or LPS $(10 \mu \mathrm{g} / \mathrm{ml})$, Pam3CSK4 $(10 \mu \mathrm{g} / \mathrm{ml})$, or CpG $(10 \mu \mathrm{g} / \mathrm{ml})$. Lysates from $1 \times 10^{7}$ red blood cells (RBCs) or pRBCs were prepared by twice freeze-thaw. After 24 hours, supernatants were collected and analysed by ELISA (eBioscience) according to manufacturer's instructions to detect IL-6 and TNF.

\section{Flow cytometry assay}

Peritoneal macrophage were isolated from mice infected with P. yoelii 17XL or $17 \mathrm{XNL}$, or from mice injected with $\mathrm{nRBCs}$, at one, three and five days after infection. $1 \times 10^{6}$ peritoneal macrophages were blocked with anti-mouse CD16/32 by incubation on ice for $30 \mathrm{~min}$, then labelled with biotin-conjugated anti-mouse TLR4 and TLR2 for 30 min, followed by incubation with PE-streptavidin for 30 
min. To examine TLR9 expression, peritoneal macrophages were permeabilized with fixation/permeabilization (eBioscience) prior to labelling with biotin-conjugated anti-mouse TLR9 and PE-streptavidin. Cells were finally analysed using a FACSCalibur flow cytometre using Cell Quest software (Becton Dickinson).

\section{Semi-quantitive reverse-transcriptase PCR}

Total RNA was isolated from $1 \times 10^{6}$ peritoneal macrophages from mice injected with $P$. yoelii 17XL or 17XNL strain, or with nRBCs, one, three and five days after infection using Trizol (Boehringer Mannheim, Germany), and reversely transcribed with MMLV (Promega, Madison, WI, USA). Initial cDNAs amount for PCR amplification were normalized with GAPDH signal, which was optimized to be visible, but not saturated. Changes in the expression of intracellular signalling molecules TRAF-6, IRAK-1, and MyD88 were investigated in peritoneal macrophages from mice infected with or without malaria parasites. Specific primers used were as follows: IRAK$1_{781}$ : 5'- GAACAGCTATCAAGGTTTCGTCA-3'; IRAK$1_{1260}$ : 5'-ACCAGCAAGGGTCTCCAGTA-3'; MyD $_{2341}$ : 5'-

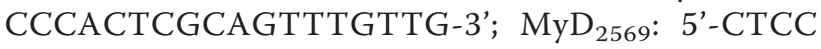
CAGTTCCTTTGTTTG-3'; TRAF 162 $_{16}$ 5'-GGGCTACGATGTGGAGTT-3'; TRAF 396 : 5'-TACCGTCAGGGAAAGAAT-3'.

\section{Statistic analysis}

Statistical significance was determined with SPSS software (version 13.0). Differences between two experimental groups (P.yoelii 17XNL-infection vs naive or P.yoelii $17 \mathrm{XL}$-infection vs naive), or two time points were analysed for statistical significance by means of a nonparametric Mann-Whitney U test, and for multiple groups (TLR2 expression among P.yoelii 17XNL-infected, P.yoelii 17XLinfected and naive mice) using Kruskal-Wallis test. ${ }^{*} P$ $<0.05$ or $* * 0<0.01$ were considered statistically significant.

\section{Results}

Plasmodium yoelii 17XNL and 17XL infection enhance the response of peritoneal macrophage to $\mathrm{PRBCs}$

Plasmodium yoelii 17XNL is a non-lethal Plasmodium strain, which grows slowly and would be cleared by mice at 20 days after infection. In contrast, $P$. yoelii $17 \mathrm{XL}$ is a lethal strain, which grows quickly and infected mice usually die at six to nine days after injection (Figure 1).

Since the macrophage-mediated innate immune response plays an important role in controlling the primary wave of $P$. yoelii infection [6], the responsiveness of macrophages collected on one, three and five days after infection with either the P. yoelii lethal 17XL strain or non-lethal 17XNL strain was investigated. Pooled adherent PECs were used as peritoneal macrophages in the following experiments without additional indication, as $85 \%$ of pooled adherent PECs were positive for the macrophage-specific marker F4/80(data not shown). Compared to the peritoneal macrophages from mice injected with $\mathrm{nRBCs}$, the reactivity of macrophages from mice infected either with $P$. yoelii 17XL or 17XNL was enhanced at one, three and five days post infection, as both inflammatory cytokines TNF and IL- 6 released by macrophages stimulated with pRBC lysate were much higher in either strain infected mice (Figure 2). However, the response of macrophages reached a peak at three days, and it was then tend to return to the baseline level at five days post $P$. yoelii 17XL-infection (Figure $2 \mathrm{~B}, \mathrm{D})$. In contrast, the response of macrophages from $P$. yoelii $17 \mathrm{XNL}$-infected mice even increased at five days post infection (Figure 2A,C). Thus, these data suggested that the response of peritoneal macrophage was enhanced after mice were infected either with $P$. yoelii $17 \mathrm{XL}$ or $17 \mathrm{XNL}$ strain, but the duration time was much longer for the macrophages from $P$. yoelii 17XNL-infected mice.

\section{Plasmodium yoelii 17XNL and 17XL infection prime the response of peritoneal macrophages to TLR agonists} It is well known that malaria parasite components, including GPI and haemozoin, could induce macrophages to release inflammatory cytokines through TLR2/4 and TLR9[8-10]. To investigate the mechanism of the enhanced response of macrophages to pRBC lysate primed by infection of P. yoelii 17XL or 17XNL, the levels of inflammatory cytokines of TNF and IL-6 released by macrophages stimulated with TLR2 agonist
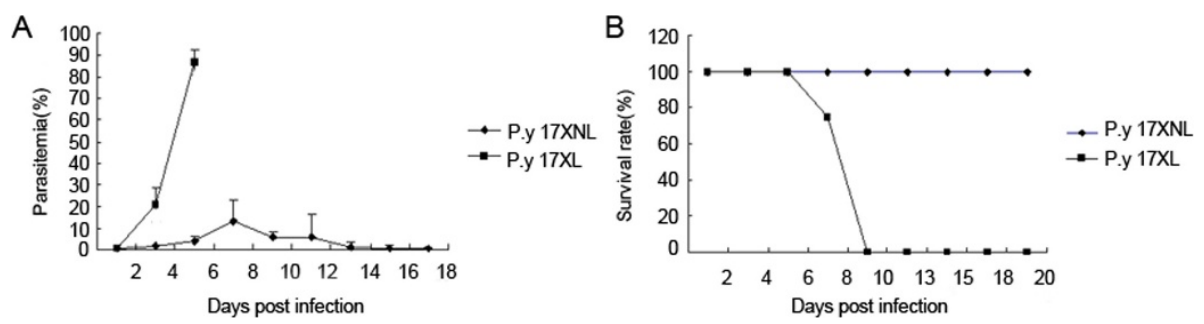

Figure 1 In vivo infection course after inoculation with $P$. y $17 \mathrm{XL}$ or $17 \mathrm{XNL}$. BALB/C were intraperitoneally injected with $2 \times 10^{5} \mathrm{P}$. yoelii $17 X L$ or $17 X N L$ parasites. Parasitaemia was recorded every other day, respectively. 


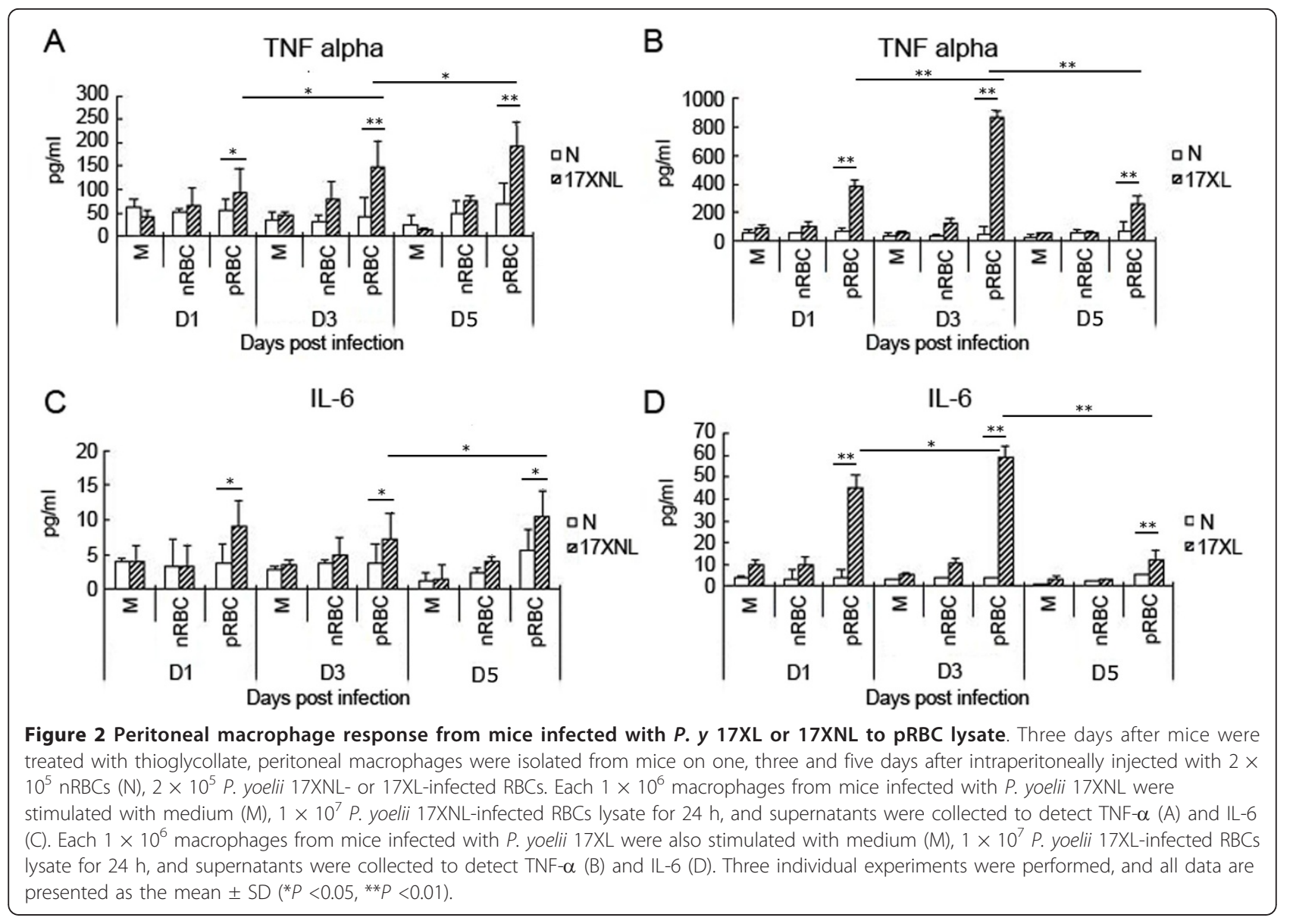

Pam3CSK4, TLR4 agonist LPS, or TLR9 agonist CpG, were measured. As shown in Figure 3, peritoneal macrophages stimulated with any of the three TLR agonists exhibited dramatically increased TNF and IL-6 levels at one, three and five days after infection with $P$. yoelii $17 \mathrm{XNL}$ (Figure 3A,C) or 17XL(Figure 3B,D), which was consistent with the response of macrophages to $\mathrm{pRBC}$ lysate. Take together, these data supported that the response of macrophages primed by $P$. yoelii $17 \mathrm{XL}$ or $17 \mathrm{XNL}$ infection was due to enhanced TLR response of macrophages.

\section{Effect of $P$. Yoelii 17XL and 17XNL infection on TLR expression of peritoneal macrophage}

It was previously reported that TLR4 and MD-2 overexpression was attributed to TLR4 signal priming induced by Propionibacterium acnes [21]. Furthermore, $P$. falciparum-induced priming of monocyte TLR response is also associated with increased expression of both TLR2 and TLR4 [15]. Hence, the expression of TLR2, TLR4, and TLR9 on peritoneal macrophages from mice infected with $P$. yoelii 17XL or 17XNL was investigated to explore the mechanism of TLR response priming induced by $P$. yoelii. As shown in Figure 4, macrophages from mice infected with $17 \mathrm{XL}$ or $17 \mathrm{XNL}$ had significantly increased expression of TLR2, but not TLR4 or TLR9, compared to the control mice at all time points measured. The strong response of macrophages from P. yoelii 17XL- or 17XNL-infected mice to TLR2 agonist Pam3CSK4 might be correlated with the relatively high level of TLR2 on PECs.

\section{Infection of $P$. Yoelii 17XL and 17XNL up-regulate TLR-} MyD88 dependent pathway intracellular molecules These data so far does not explain the underlying mechanism of the primed TLR4 and TLR9 responses of macrophages induced by $P$. yoelii $17 \mathrm{XL}$ and $17 \mathrm{XNL}$ infection[15]. Since p38 activity enhancement are related to TLR response priming in malaria parasite infection [16], then the transcription of MyD88, IRAK-1, and TRAF6, which are shared by the TLR2-, TLR4-, and TLR9- mediated MyD88-dependent pathway, were investigated in macrophages from $P$. yoelii $17 \mathrm{XL}$ - or $17 \mathrm{XNL}$-infected mice. As shown in Figure 5, the transcriptional levels of MyD88, IRAK-1, and TRAF-6 were significantly increased in mice infected with either $P$. 


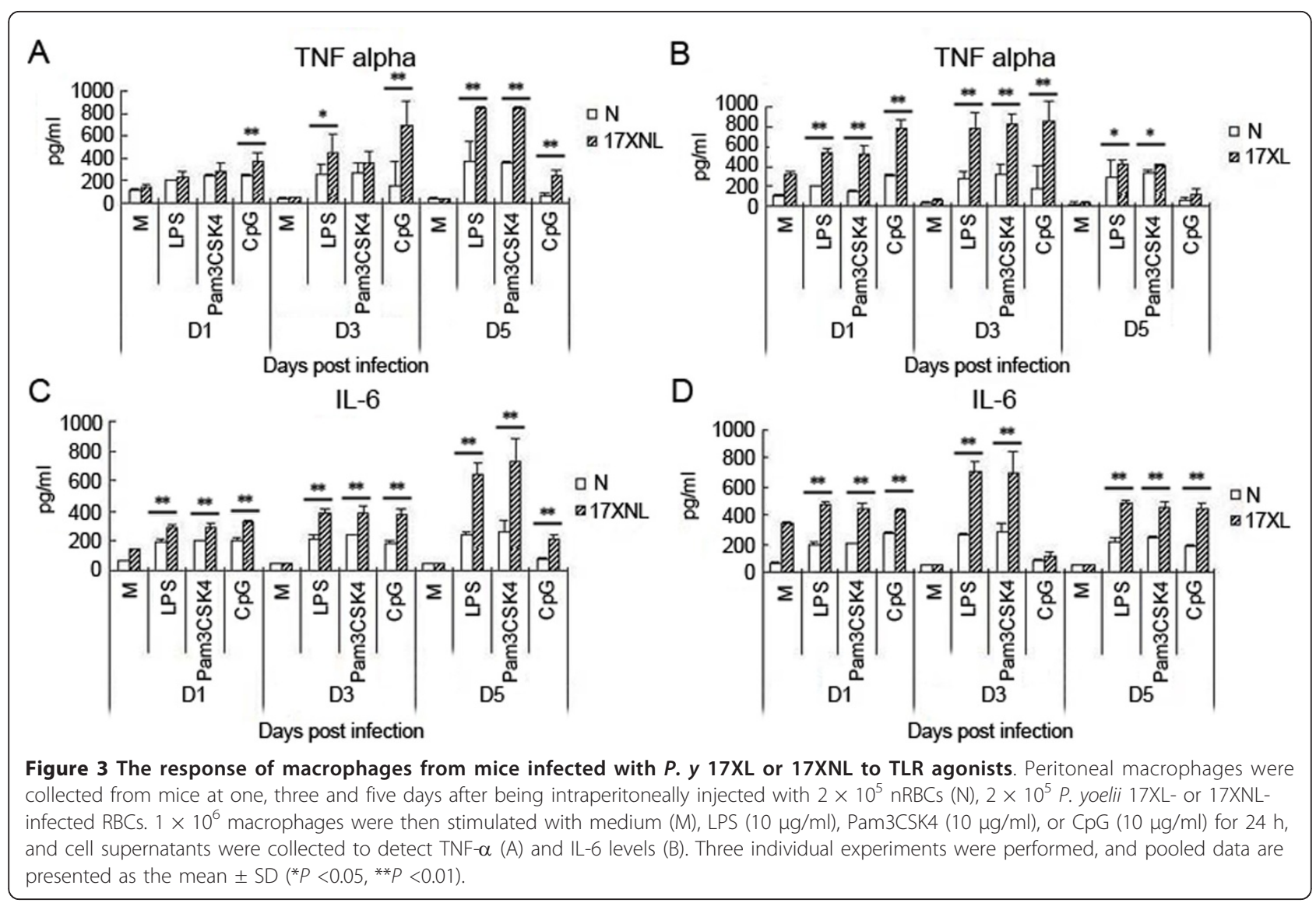

yoelii 17XL or 17XNL compared to control mice at one, three and five days post-infection. The change pattern of transcription levels of MyD88, IRAK-1, and TRAF-6 of macrophage was consistent with its response to $\mathrm{pRBC}$ lysate after infection with either strain. These data strongly suggested an important role for the up-

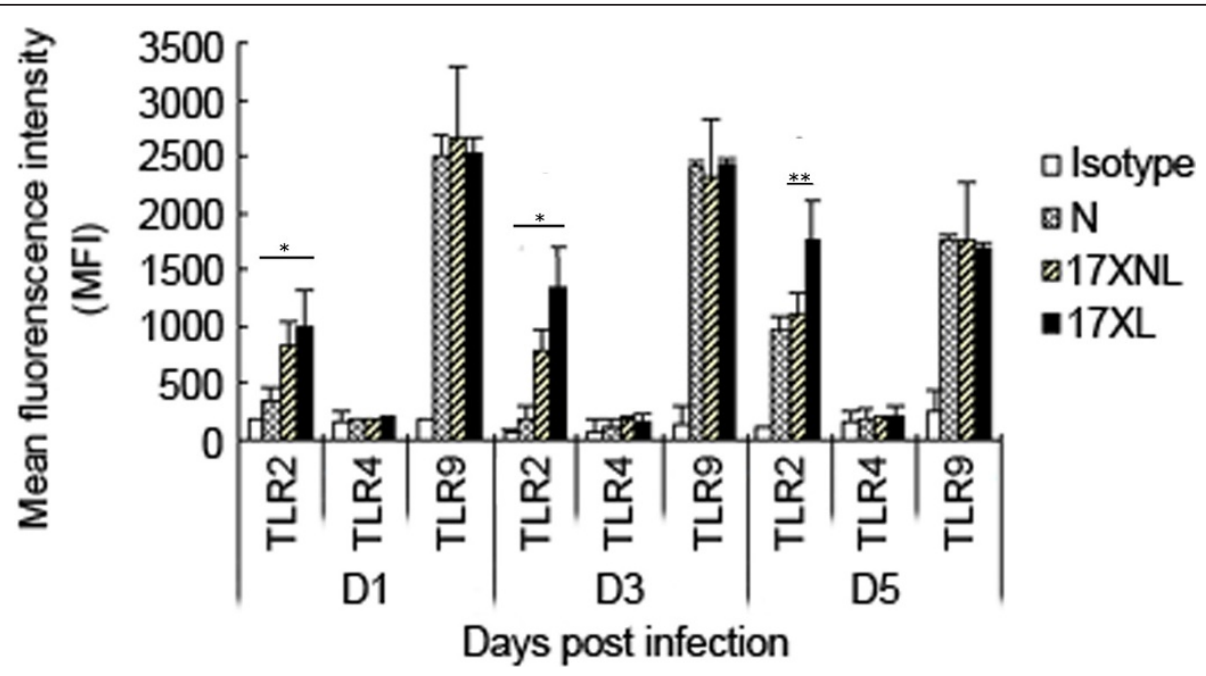

Figure 4 TLR2, TLR4 and TLR9 expression on macrophages from mice infected with $P$. y 17XL or 17XNL. Peritoneal macrophages were collected from mice at one, three and five days after they had been intraperitoneally injected with $\mathrm{nRBC}$, $P$. yoelii $17 \mathrm{XL}$ - or $17 \mathrm{XNL}$-infected RBCs. Macrophages were labelled with anti-mouse TLR4, TLR2 and TLR9, and analysed on a FACSCalibur using Cell Quest software. Data are presented as the mean fluorescence intensity $(\mathrm{MFI})$, and values are shown as mean $\pm \mathrm{SD}\left({ }^{*} P<0.05,{ }^{* *} P<0.01\right)$. 


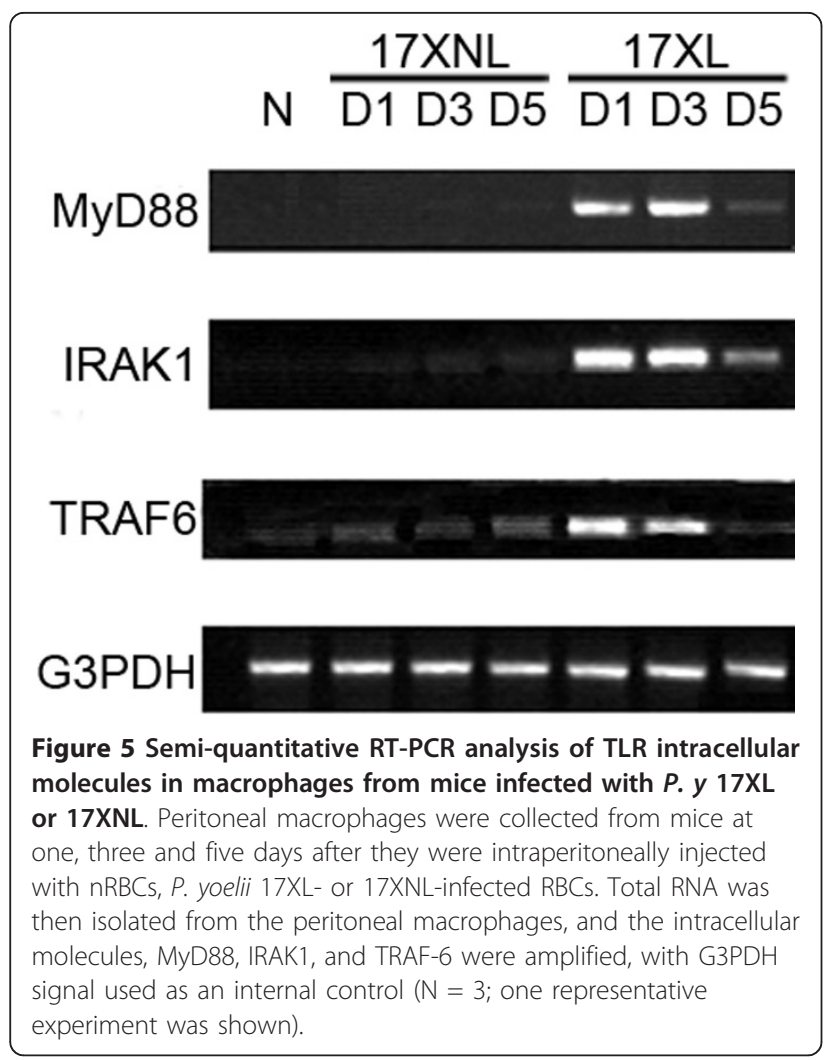

regulation of MyD88, IRAK-1, and TRAF-6 in the TLR response priming induced by either $P$. yoelii strain infection.

\section{Discussion}

Macrophage-mediated innate immune response is critical for controlling the $P$. yoelii parasitaemia at its early stage, so it tends to be modulated by exposure to malaria parasites. It was previously reported that Plasmodium berghei infection inhibits IL-12 p40 production by peritoneal macrophages at the transcriptional level [22]. However, infection with lethal strain P. yoelii 17XL or non-lethal strain $P$. yoelii $17 \mathrm{XNL}$ was found to be able to prime the response of macrophages through upregulating TLR2 expression and signalling intracellular molecules, although infection of the two strains resulted in dramatically different disease outcome.

Pre-exposure to a variety of TLR agonists, including LPS, Pam3CSK4, and CpG, often induces macrophages into a tolerant status to prevent over-activation [23-25]. It is well known that pre-administration of a low dose (sub-lethal dose) of LPS induces macrophages into endotoxin tolerance to protect the host from the challenge of lethal-dose of LPS. However, tissue injury[26] and Propionibacterium infection [21] have been demonstrated to prime the TLR response. In this study, the lethal $P$. yoelii 17XL strain was also found to enhance the response of murine peritoneal macrophages to $\mathrm{pRBC}$ at one, there and five days post-infection (Figure 3B-D), which is consistent with recent reports of priming the TLR response with $P . f$ at the early stage [15-17]. Interestingly, infection with the non-lethal $P$. yoelii $17 \mathrm{XNL}$ strain could also prime the response of macrophages to pRBC lysate (Figure 3A-C). Like the response of macrophages to $\mathrm{pRBC}$ lysate, macrophages from $P$. yoelii $17 \mathrm{XL}$ - or $17 \mathrm{XNL}$-infected mice responded to TLR2, TLR4, and TLR9 agonists much more strongly than macrophages from nRBCs-injected mice at one and there days post-infection (Figure 4). Thus, the increased response of macrophages to TLR2, TLR4, and TLR9 agonists resulted in their hypersensitivity to pRBC lysate.

In a previous study, McCall et al attempted to correlate TLR2/4 expression with the priming response, however the investigators did not observe enhanced expression of TLR2/4 on PBMCs [17]. In contrast, Flanklin et al found that TLR2, TLR4 and TLR9 expression was significantly augmented in PBMCs from patients with relatively high parasitaemia [15]. Here, the infection with $P$. yoelii 17XL or 17XNL induced the expression of TLR2, but not TLR4 and TLR9, on murine macrophages in the present study (Figure 5). The disparity could be interpreted as $P$. yoelii used in this study, but $P . f$ was used in their research. Interestingly, the transcription levels of intracellular molecules of the MyD88-dependent pathway were also found to be augmented in macrophages from $P$. yoelii $17 \mathrm{XL}$ - or $17 \mathrm{XNL}$ infected mice (Figure 5). Therefore, hypersensitivity of macrophages to TLR agonists was contributed to upregulation of intracellular signalling molecules by malaria parasite infection. However, it remains to be determined whether up-regulation of MyD88, IRAK-1, and TRAF- 6 would result in enhancement of MAPK activation, which was previously contributed to priming the TLR response on PBMCs from P. f-infected patients [16].

It was recently reported that malaria-induced priming of the TLR response was TLR9-, MyD88-, and IFN- $\gamma-$ dependent[15]. Hence, it is reasonable to find that lethal and non-lethal strains can prime the macrophage response in this study, as a relative high level of IFN- $\gamma$ is induced in the spleen of either strain-infected mice during the early stage [27]. It is well known that IFN- $\gamma$ was mainly secreted by NK and T cells after infection with lethal strain P. yoelii 17XNL and nonlethal strain P. yoelii 17XL [4], but the production of IFN- $\gamma$ by NK cells required the help of IL-12 of DC activated by rodent malaria parasite [28].

Single amino acid substitution of erythrocytic binding ligand (EBL) was reported to determine the erythrocyte invasion preference and virulence of $P$. yoelii $17 \mathrm{XL}$ and $P$. yoelii 17XNL [29], but the early induction of TGF- $\beta$ 
[30] and activation of $\mathrm{CD} 4^{+} \mathrm{CD} 25^{+} \mathrm{T}$ cells could suppress the host immune response, and result in the overgrowth of $P$. yoelii 17XL in mice. Although the level of macrophage response between the two strains could not be compared in this study, as their parasitaemia were significantly different at one, there and five days post infection (Figure 1), the duration time of primed TLRs response of macrophage from $P$. yoelii 17XNL-infected mice was much longer than that from $P$. yoelii $17 \mathrm{XL}-$ infected mice. This is consistent with a relative higher level of IFN- $\gamma$ in the spleen of $P$. yoelii 17XNL-infected mice than that of $P$. yoelii 17XL-infected mice [27], and might be associated with more efficiently controlling of $P$. yoelii $17 \mathrm{XNL}$ growth than $P$. yoelii $17 \mathrm{XL}$ in mice at the early stage.

\section{Conclusion}

It was observed that $P . y$, either its lethal $17 \mathrm{XL}$ strain or non-lethal 17XNL strain, primes the TLR response on macrophages, mainly through modulating the transcription of intracellular signalling molecules. However, the enhanced macrophage response induced by $P$. yoelii 17XNL maintained longer than that induced by lethal $P$. yoelii 17XL. This finding provides us with a novel aspect of TLR response modulated by malaria parasites with different virulence, and clues to understand the control mechanism of the primary wave of Plasmodium yoelii.

\section{Abbreviations}

DC: Dendritic cell; EBL: Erythrocytic binding ligand; GPI:

Glycosylphosphatidylinositols; LPS: Lipopolysaccharide; MFI: Mean fluorescence intensity; PBMC: Peripheral blood mononuclear cells; MAPK: Mitogen-activated protein kinase; RBCs: Red blood cells; nRBCs: Normal red blood cells; PECs: Peritoneal exudate cells; pRBCs: Parasitized red blood cells; P. chabaudi: Plasmodium chabaudi; P. yoelii: Plasmodium yoelii; P. falciparum: Plasmodium falciparum; TLRs: Toll-like receptors.

\section{Acknowledgements}

We thank W Peters and B L Robinson for providing Plasmodium yoelii $17 \mathrm{XL}$ to the Malaria Research and Reference Reagent Resource Center, and thank Professor Kaifa Wang for his assistance for stastistical analysis. This work is supported by National Basic Research Program of China, 973 Program 2007CB513105, National Natural Science Foundation of China (30972773), and Natural Science Foundation Project of CQ CSTC (2008BA5010).

\section{Author details}

'Department of Pathogenic Biology, Third Military Medical University, 30 Gaotanyan Zhengjie, Shapingba District, Chongqing 400038, People's Republic of China. ${ }^{2}$ Institute of Immunology, PLA, Third Military Medical University, 30 Gaotanyan Zhengjie, Shapingba District, Chongqing 400038, People's Republic of China.

\section{Authors' contributions}

WYX designed research; YF, YD, TLZ, XLF performed research; WYX analysed data and wrote paper. All authors have read and approved the final manuscript.

\section{Competing interests}

The authors declare that they have no competing interests.

Received: 4 January 2012 Accepted: 1 April 2012 Published: 1 April 2012
References

1. WHO: World Malaria Report 2009 [http://www.who.int/malaria/ world_malaria_report_2009/en/index.html].

2. Good MF, Xu H, Wykes M, Engwerda CR: Development and regulation of cell-mediated immune responses to the blood stages of malaria: implications for vaccine research. Annu Rev Immunol 2005, 23:69-99.

3. Mannoor MK, Halder RC, Morshed SR, Ariyasinghe A, Bakir HY, Kawamura H, Watanabe $H$, Sekikawa $H$, Abo T: Essential role of extrathymic T cells in protection against malaria. J Immunol 2002, 169:301-306.

4. Choudhury HR, Sheikh NA, Bancroft GJ, Katz DR, De Souza JB: Early nonspecific immune responses and immunity to blood-stage nonlethal Plasmodium yoelii malaria. Infect Immun 2000, 68:6127-6132.

5. Mohan K, Moulin P, Stevenson MM: Natural killer cell cytokine production, not cytotoxicity, contributes to resistance against blood-stage Plasmodium chabaudi AS infection. J Immunol 1997, 159:4990-4998.

6. Couper KN, Blount DG, Hafalla JC, van Rooijen N, de Souza JB, Riley EM: Macrophage-mediated but gamma interferon-independent innate immune responses control the primary wave of Plasmodium yoelii parasitemia. Infect Immun 2007, 75:5806-5818.

7. Freitas do Rosario AP, Voisine C, Mastelic B, Thompson J, Koernig S, Jarra W, Renia L, Mauduit M, Potocnik AJ, Langhorne J, Sponaas AM: Migrating monocytes recruited to the spleen play an important role in control of blood stage malaria. Blood 2009, 114:5522-5531.

8. Zhu J, Krishnegowda G, Gowda DC: Induction of proinflammatory responses in macrophages by the glycosylphosphatidylinositols of Plasmodium falciparum: the requirement of extracellular signal-regulated kinase, p38, c-Jun N-terminal kinase and NF-kappaB pathways for the expression of proinflammatory cytokines and nitric oxide. J Biol Chem 2005, 280:8617-8627.

9. Krishnegowda G, Hajjar AM, Zhu J, Douglass EJ, Uematsu S, Akira S, Woods AS, Gowda DC: Induction of proinflammatory responses in macrophages by the glycosylphosphatidylinositols of Plasmodium falciparum: cell signaling receptors, glycosylphosphatidylinositol (GPI) structural requirement, and regulation of GPI activity. J Biol Chem 2005, 280:8606-8616

10. Coban C, Ishii KJ, Kawai T, Hemmi H, Sato S, Uematsu S, Yamamoto M, Takeuchi O, Itagaki S, Kumar N, Horii T, Akira S: Toll-like receptor 9 mediates innate immune activation by the malaria pigment hemozoin. $J$ Exp Med 2005, 201:19-25.

11. Parroche $P$, Lauw FN, Goutagny N, Latz E, Monks BG, Visintin A, Halmen KA, Lamphier M, Olivier M, Bartholomeu DC, Gazzinelli RT, Golenbock DT: Malaria hemozoin is immunologically inert but radically enhances innate responses by presenting malaria DNA to Toll-like receptor 9. Proc Natl Acad Sci USA 2007, 104:1919-1924.

12. Wu X, Gowda NM, Kumar S, Gowda DC: Protein-DNA complex is the exclusive malaria parasite component that activates dendritic cells and triggers innate immune responses. J Immunol 2010, 184:4338-4348.

13. Nomura F, Akashi S, Sakao Y, Sato S, Kawai T, Matsumoto M, Nakanishi K, Kimoto M, Miyake K, Takeda K, Akira S: Cutting edge: endotoxin tolerance in mouse peritoneal macrophages correlates with down-regulation of surface toll-like receptor 4 expression. J Immunol 2000, 164:3476-3479.

14. Medvedev AE, Lentschat A, Wahl LM, Golenbock DT, Vogel SN: Dysregulation of LPS-induced Toll-like receptor 4-MyD88 complex formation and IL-1 receptor-associated kinase 1 activation in endotoxintolerant cells. J Immunol 2002, 169:5209-5216.

15. Franklin BS, Parroche $P$, Ataíde MA, Lauw F, Ropert C, de Oliveira RB, Pereira D, Tada MS, Nogueira P, da Silva LH, Bjorkbacka H, Golenbock DT, Gazzinelli RT: Malaria primes the innate immune response due to interferon-gamma induced enhancement of toll-like receptor expression and function. Proc Natl Acad Sci USA 2009, 106:5789-5794.

16. Hartgers FC, Obeng BB, Voskamp A, Larbi IA, Amoah AS, Luty AJ, Boakye D, Yazdanbakhsh M: Enhanced Toll-like receptor responsiveness associated with mitogen-activated protein kinase activation in Plasmodium falciparum-infected children. Infect Immun 2008, 76:5149-5157.

17. McCall MB, Netea MG, Hermsen CC, Jansen T, Jacobs L, Golenbock D, van der Ven AJ, Sauerwein RW: Plasmodium falciparum infection causes proinflammatory priming of human TLR responses. J Immunol 2007, 179:162-171.

18. Perry JA, Olver CS, Burnett RC, Avery AC: Cutting edge: the acquisition of TLR tolerance during malaria infection impacts $T$ cell activation. $J$ Immunol 2005, 174:5921-5925. 
19. Landau I, Chabaud AG: Natural infection by 2 plasmodia of the rodent Thamnomys rutilans in the Central African Republic. Comptes Rendus Hebdomadaires des Seances de l'Academie des Sciences. D: Sciences Naturelles $1965,261: 230-232$

20. Yoeli M, Hargreaves B, Carter R, Walliker D: Sudden increase in virulence in a strain of Plasmodium berghei yoelii. Ann Trop Med Parasitol 1975, 69:173-178.

21. Romics L Jr, Dolganiuc A, Kodys K, Drechsler Y, Oak S, Velayudham A, Mandrekar P, Szabo G: Selective priming to Toll-like receptor 4 (TLR4), not TLR2, ligands by $P$. acnes involves up-regulation of MD- 2 in mice. Hepatology 2004, 40:555-564.

22. Xu X, Sumita K, Feng C, Xiong X, Shen H, Maruyama S, Kanoh M, Asano Y: Down-regulation of IL-12 p40 gene in Plasmodium berghei-infected mice. J Immunol 2001, 167:235-241.

23. Medvedev AE, Kopydlowski KM, Vogel SN: Inhibition of lipopolysaccharideinduced signal transduction in endotoxin-tolerized mouse macrophages: dysregulation of cytokine, chemokine, and toll-like receptor 2 and 4 gene expression. J Immunol 2000, 164:5564-5574.

24. Siedlar M, Frankenberger M, Benkhart E, Espevik T, Quirling M, Brand K, Zembala M, Ziegler-Heitbrock L: Tolerance induced by the lipopeptide Pam3Cys is due to ablation of IL-1R-associated kinase-1. J Immunol 2004, 173:2736-2745.

25. Yeo SJ, Yoon JG, Hong SC, Yi AK: CpG DNA induces self and crosshyporesponsiveness of RAW264.7 cells in response to CpG DNA and lipopolysaccharide: alterations in IL-1 receptor-associated kinase expression. J Immunol 2003, 170:1052-1061.

26. Paterson HM, Murphy TJ, Purcell EJ, Shelley O, Kriynovich SJ, Lien E, Mannick JA, Lederer JA: Injury primes the innate immune system for enhanced Toll-like receptor reactivity. J Immunol 2003, 171:1473-1483.

27. De Souza JB, Williamson KH, Otani T, Playfair JH: Early gamma interferon responses in lethal and nonlethal murine blood-stage malaria. Infect Immun 1997, 65:1593-1598.

28. Ing R, Stevenson MM: Dendritic cell and NK cell reciprocal cross talk promotes gamma interferon-dependent immunity to blood-stage Plasmodium chabaudi AS infection in mice. Infect Immun 2009, 77:770-782.

29. Otsuki H, Kaneko O, Thongkukiatkul A, Tachibana M, Iriko H, Takeo S, Tsuboi T, Torii M: Single amino acid substitution in Plasmodium yoelii erythrocyte ligand determines its localization and controls parasite virulence. Proc Natl Acad Sci USA 2009, 106:7167-7172.

30. Omer FM, de Souza JB, Riley EM: Differential induction of TGF-beta regulates proinflammatory cytokine production and determines the outcome of lethal and nonlethal Plasmodium yoelii infections. J Immunol 2003, 171:5430-5436.

doi:10.1186/1475-2875-11-104

Cite this article as: Fu et al:: Plasmodium yoelii blood-stage primes macrophage-mediated innate immune response through modulation of toll-like receptor signalling. Malaria Journal 2012 11:104.

\section{Submit your next manuscript to BioMed Central and take full advantage of:}

- Convenient online submission

- Thorough peer review

- No space constraints or color figure charges

- Immediate publication on acceptance

- Inclusion in PubMed, CAS, Scopus and Google Scholar

- Research which is freely available for redistribution

Submit your manuscript at www.biomedcentral.com/submit
Biomed Central 